\title{
Nitric Oxide Produced by Ultraviolet-irradiated Keratinocytes Stimulates Melanogenesis
}

\author{
Christine Roméro-Graillet, Edith Aberdam, Monique Clément, Jean-Paul Ortonne, and Robert Ballotti
}

Institut National de la Santé et de la Recherche Médicale U385, Faculté de Médecine, 06107 Nice cedex 02, France

\begin{abstract}
Ultraviolet (UV) radiation is the main physiological stimulus for human skin pigmentation. Within the epidermalmelanin unit, melanocytes synthesize and transfer melanin to the surrounding keratinocytes. Keratinocytes produce paracrine factors that affect melanocyte proliferation, dendricity, and melanin synthesis. In this report, we show that normal human keratinocytes secrete nitric oxide (NO) in response to UVA and UVB radiation, and we demonstrate that the constitutive isoform of keratinocyte NO synthase is involved in this process. Next, we investigate the melanogenic effect of NO produced by keratinocytes in response to UV radiation using melanocyte and keratinocyte cocultures. Conditioned media from UV-exposed keratinocytes stimulate tyrosinase activity of melanocytes. This effect is reversed by NO scavengers, suggesting an important role for NO in UVinduced melanogenesis. Moreover, melanocytes respond to NO-donors by decreased growth, enhanced dendricity, and melanogenesis. The rise in melanogenesis induced by NOgenerating compounds is associated with an increased amount of both tyrosinase and tyrosinase-related protein 1 .

These observations suggest that NO plays an important role in the paracrine mediation of $\mathrm{UV}$-induced melanogenesis. (J. Clin. Invest. 1997. 99:635-642.) Key words: nitric oxide - ultraviolet light • keratinocytes • secreted factor • pigmentation
\end{abstract}

\section{Introduction}

The melanin pigmentary system of the human skin is based on two cell types, melanocytes and keratinocytes, interacting within a functional unit, the epidermal-melanin unit (1). The functional activity of this unit, rather than the melanocyte alone, is the focal point for the determination of skin color. It now appears that keratinocytes, as well as receiving melanin, may also regulate both growth and differentiation of melanocytes (2-5). Ultraviolet (UV) radiation is the main stimulus for skin pigmentation; in vivo, it increases proliferation and melanization of melanocytes (6). To stimulate melanogenesis, UV radiation can act directly on cultured melanocytes (7-9) or indirectly through the release of keratinocyte-derived factors $(10-15)$. Production of basic fibroblast growth factor, endothe-

Address correspondence to Christine Roméro-Graillet, INSERM U385, Faculté de Médecine, Avenue de Valombrose, 06107 Nice cedex 02, France. Phone: 330493 377 790; FAX: 330493811 404; E-mail: romero@unice.fr

Received for publication 1 July 1996 and accepted in revised form 9 December 1996.

J. Clin. Invest.

(C) The American Society for Clinical Investigation, Inc.

0021-9738/97/02/0635/08 \$2.00

Volume 99, Number 4, February 1997, 635-642 lin-1 (ET-1), ${ }^{1}$ and GM-CSF by keratinocytes is upregulated after UV light exposure, and these peptides stimulate melanocyte growth (16-19). In addition, ET-1 regulates human melanocytes' melanization via a receptor-mediated signal transduction pathway (20). However, the concentration of ET-1 in keratinocyte-conditioned media is considerably lower than the concentration of ET-1 required to elicit a melanogenic effect (0.5 pM vs. $10 \mathrm{nM})$, suggesting that other soluble melanogenic mediators are present in keratinocyte-conditioned media (20).

Interestingly, nitric oxide (NO) that is a diffusible free radical, displaying pleiotropic bioregulatory effects in diverse cells and tissues $(21,22)$ has been reported to be produced by melanocytes (23) and keratinocytes in response to proinflammatory cytokines $(24,25)$. Moreover, NO contributes to erythema, in response to UVB light, by increasing blood flow in human skin microcirculation (26). Thus, NO appears to be a potential UV mediator acting within the epidermal melanin unit. NO is synthesized by the enzyme NO synthase (NOS). Three NOSs were identified by cloning of their complementary and genomic DNAs (27-29). One isoform seems confined to endothelium (ecNOS type III), while another one is largely but not exclusively expressed in peripheral and central neurons (cNOS type I). The third isoform (iNOS type II) is absent in most cells under normal conditions, but it can be induced in several cell types by stress signals from the immune and inflammatory systems $(30,31)$.

In the present report, we first studied the effect of UVA and UVB on the production of NO by normal human keratinocytes. We found that UVA and UVB induce production of NO through the activation of cNOS type I. We showed that in presence of NO scavengers, the stimulatory effect on melanogenesis observed in the presence of irradiated keratinocytes is almost completely reversed. Additionally, we demonstrated that NO donors increase tyrosinase activity and melanin synthesis in human melanocytes. This effect is positively correlated with an increased amount of both tyrosinase and tyrosinase-related protein 1 (TRP-1), two enzymes involved in melanogenesis $(8,32)$. Taken together, these findings support the hypothesis that NO is involved in the paracrine mediation of UV-induced melanogenesis.

\section{Methods}

\section{Chemicals}

$\mathrm{N}$-monomethyl-L-arginine (L-NMA), S-nitroso- $\mathrm{N}$-acetylpenicillamine (SNAP), and 2 phenyl,4,4,5,5 tetramethylimidazoline, 1oxyl, 3oxide (carboxy-PTIO) were from Affiniti (Nottingham, UK). The (6R)5,6,7,8-tetrahydro-L-biopterin dihydrochloride was from INC (Orsay,

1. Abbreviations used in this paper: carboxy-PTIO, 2 phenyl,4,4,5,5 tetramethylimidazoline, 1oxyl, 3oxide; cNOS, constitutive NOS; ET-1, endothelin-1; Hb, hemoglobin; iNOS, inducible NOS; L-NMA, $N$-monomethyl-L-arginine; NO, nitric oxide; NOS, NO synthase, SNAP, $\mathrm{S}$-nitroso- $N$-acetylpenicillamin; SNP, sodium nitroprusside; TRP-1, tyrosinase-related protein 1. 
France). FMN, calmodulin, b-NADPH, sodium nitroprusside (SNP), and hemoglobin $(\mathrm{Hb})$, were obtained from Sigma Chemical Co. (La Verpillère, France). L-[2-3-4-5- $\left.{ }^{3} \mathrm{H}\right]$ arginine $(58 \mathrm{Ci} / \mathrm{mmol}, 1 \mathrm{mCi} / \mathrm{ml})$ was from Amersham Laboratories (Les Ulis, France). All other products used for cell culture and biochemical analysis were obtained as previously reported $(8,33)$.

\section{Cell culture}

Human melanocyte cultures were initiated from neonatal foreskins of caucasoid individuals and grown according to a modified method of Eisinger and Marko (34) as previously described (8). $2 \mathrm{~d}$ before experiments, complete melanocyte growth medium was removed and replaced by minimum growth medium consisting of MCDB 153 containing $2 \%$ fetal calf serum, $5 \mu \mathrm{g} / \mathrm{ml}$ insulin, and $30 \mu \mathrm{g} / \mathrm{ml}$ bovine pituitary extract.

Human keratinocytes from neonatal foreskin were initiated into culture by the method of Rheinwald and Green (35). The cells were subcultured and passaged in serum-free medium (KDM; PromoCell, Heidelger), according to Boyce and Ham (36) as modified by O'Keefe and Chiu (37).

For coculture experiments, melanocyte cultures were obtained by plating $5 \times 10^{5}$ cells onto 35 -mm tissue culture multi wells (at the lower compartment of the melanocyte-keratinocyte coculture). Keratinocytes were seeded $\left(5 \times 10^{5}\right.$ cells $\left./ 24.5 \mathrm{~mm}\right)$ onto polycarbonate filters (Transwell; Costar, France), corresponding to the upper compartment of the melanocyte-keratinocyte coculture. During phototreatment, melanocyte-keratinocyte cocultures were grown in a mixture of KDM:minimal melanocyte growth medium (ratio 1:1). For control, melanocytes and keratinocytes alone were identically handled.

\section{Ultraviolet irradiation}

The source of ultraviolet radiation was a Vilber Lourmat stimulator (Marne la Vallée, France) fitted out with an ultraviolet (UV) A irradiation source composed of Vilber Lourmat tubes T-20.L-365 (no UVB, no UVC emission), a UVB irradiation source composed of Vilber Lourmat tubes T-20.M-312 (no UVA, no UVC emission), mercury vapor tubes, low pressure, hot cathodes with a Vilber Lourmat RMX-365/312 radiometer with microprocessor programmable in energy (millijoules/square centimeter) with time basis enabling six irradiation measurements per second for controlling the energy received by the sample. Keratinocytes were irradiated daily for $4 \mathrm{~d}$ with 300 $\mathrm{mJ} / \mathrm{cm}^{2}$ of UVA or $50 \mathrm{~mJ} / \mathrm{cm}^{2}$ of UVB. Nonirradiated keratinocytes were shielded from UV radiation by covering wells with taped lids. All cells were handled at room temperature during the time of whole photo treatment. The UVA and UVB doses used in this study correspond to 90-s and 5-min exposures to the midday sunlight in a highly sunny area like Arizona, where the irradiance of UVA and UVB radiations from the sunlight are, respectively, 6 and $0.2 \mathrm{~mW} / \mathrm{cm}^{2}$ (38). $24 \mathrm{~h}$ after the last irradiation, the cell viability was estimated by the Trypan blue exclusion method. After UVA and UVB treatments, we observed, respectively, $10 \pm 3.5$ and $14 \pm 2 \%$ of dead cells (The numbers represent the mean of four dishes $\pm \mathrm{SE}$ ).

NO is an extremely labile free radical with a very short half life $(5 \mathrm{~s}$ $-4 \mathrm{~min}$ ); the duration of the irradiations is $3 \mathrm{~min}$ for UVA, $300 \mathrm{~mJ} /$ $\mathrm{cm}^{2}$, and $90 \mathrm{~s}$ for UVB, $50 \mathrm{~mJ} / \mathrm{cm}^{2}$. Thus, we have chosen to irradiate the melanocyte-keratinocyte cocultures in culture medium in order to avoid any loss of NO produced by UV-exposed keratinocytes during the manipulation. Nevertheless, we have evaluated the melanogenic potential of irradiated media by UVA and UVB lights on melanocytes. Irradiated media were not able to promote melanogenesis in these cells and had no effect on melanocyte proliferation.

\section{NO donors treatment}

To evaluate the effect of $\mathrm{NO}$ on melanogenesis, normal human melanocytes were incubated in the presence of different chemical NO donors. Melanocytes were treated for $4 \mathrm{~d}$ in the presence of SNP (readily released) or SNAP $\left(t_{1 / 2}=4.6 \mathrm{~h}\right)$. 24-h-old solutions of NO donors (which only contain the inactive by-products) were used as control. Trypan blue exclusion method was used for cell viability estimation. Thus, treatments with SNAP 100, 200, and $400 \mu \mathrm{M}$ induced, respectively, $8 \pm 4,15 \pm 3$, and $30 \pm 5 \%$ of melanocyte death, and treatments with 50,100 , and $200 \mu \mathrm{M}$ of SNP induced, respectively, $10 \pm 2$, $13 \pm 3$, and $21 \pm 4 \%$ of cell death (mean of four dishes $\pm \mathrm{SE}$ ).

\section{Measurement of nitrite}

To measure nitrite production by human keratinocytes, keratinocyteconditioned media were collected $24 \mathrm{~h}$ after the last irradiation, centrifuged (15 $\mathrm{min}, 1,200 \mathrm{rpm}$ ) and $0.2-\mu \mathrm{m}$ filtered to remove cellular debris. Nitrite concentration was estimated by a spectrophotometric method based on the Greiss reaction as described by Wang et al. (39).

\section{Image analysis}

Morphometric measurements were performed using a Biocom 500 (BIOCOM SA, Les Ulis, France) image analysis system coupled to a CCD video camera and a TMS inverted light microscope (Nikon Inc., Melville, NY). After treatment, cells were viewed using a $20 \times$ phase contrast objective and projected onto the video screen. Outlines of 100 cells of each experimental condition were acquired manually, quantitative measurements of maximal cell length were performed using the Mima software.

\section{Enzymatic assays}

All results were normalized to cell number or to protein amount/ assay dish using a protein kit assay (Bio-Rad Laboratories, Hercules, CA).

\section{Measurement of NOS activity}

NOS activity was defined as the L-NMA $(1 \mathrm{mM})$ inhibitable rate of conversion of L-[2-3-4-5- $\left.{ }^{3} \mathrm{H}\right]$ arginine to $\mathrm{L}-\left[2-3-4-5-{ }^{3} \mathrm{H}\right]$ citrulline by cell lysates according to an adaptation (40) of the method of Evans et al. (41).

\section{Melanogenic activity assays}

Tyrosinase activity in living cells (tyrosinase activity in situ). The early rate-limiting step of the biosynthetic pathway of melanin (hydroxyla-
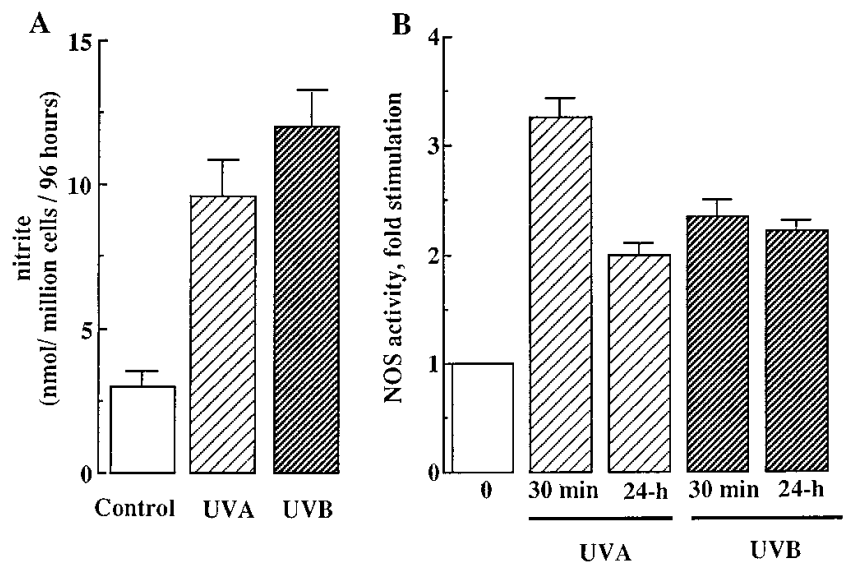

Figure 1. Production of nitric oxide by keratinocytes. $(A)$ Effects of UV exposure on the secretion of nitrite into culture medium. Cells grown in KDM were treated daily with UVA $\left(4 \times 300 \mathrm{~mJ} / \mathrm{cm}^{2}\right)$ or UVB $\left(4 \times 50 \mathrm{~mJ} / \mathrm{cm}^{2}\right)$. The accumulation of $\mathrm{NO}$ in the media collected after $4 \mathrm{~d}$ of culture was assayed. Nitrite content is expressed as $\mathrm{nmol} / 10^{6}$ cells. Each point represents the mean of three samples \pm SE. (B) Activity of keratinocyte NO synthase after a single UV exposure. Cells were exposed once to UVA $\left(300 \mathrm{~mJ} / \mathrm{cm}^{2}\right)$ or UVB light $(50 \mathrm{~mJ} /$ $\left.\mathrm{cm}^{2}\right)$, and then harvested at the indicated time. Results express the L-NMA $(1 \mathrm{mM})$ inhibitable rate of L-arginine into L-citrulline as described in the experimental procedure section. In the presence of L-NMA, NO synthase basal activity is $69 \pm 6 \mathrm{pmol} / \mathrm{min}$ per $\mathrm{mg}$ protein. Each point represents the mean of three samples \pm SE of one representative experiment. 


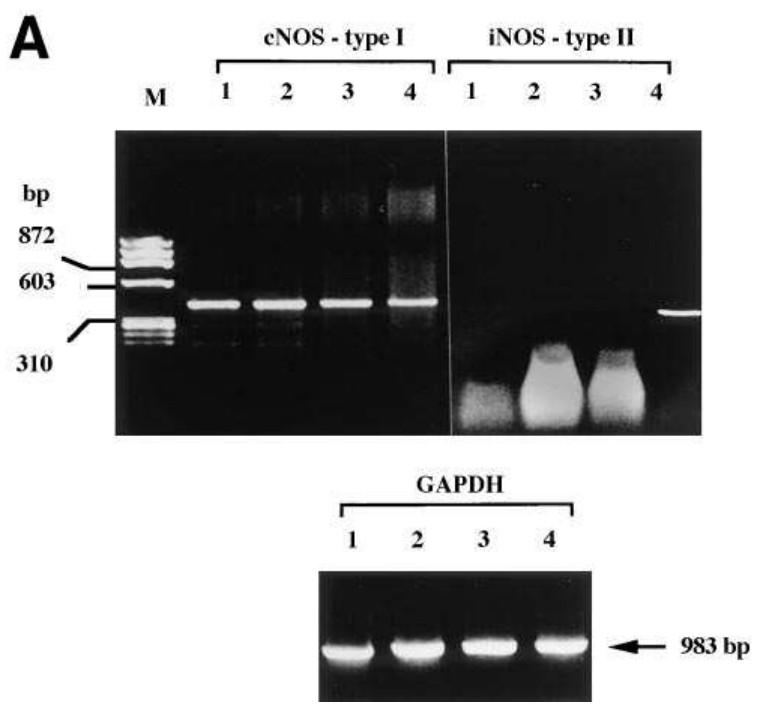

B
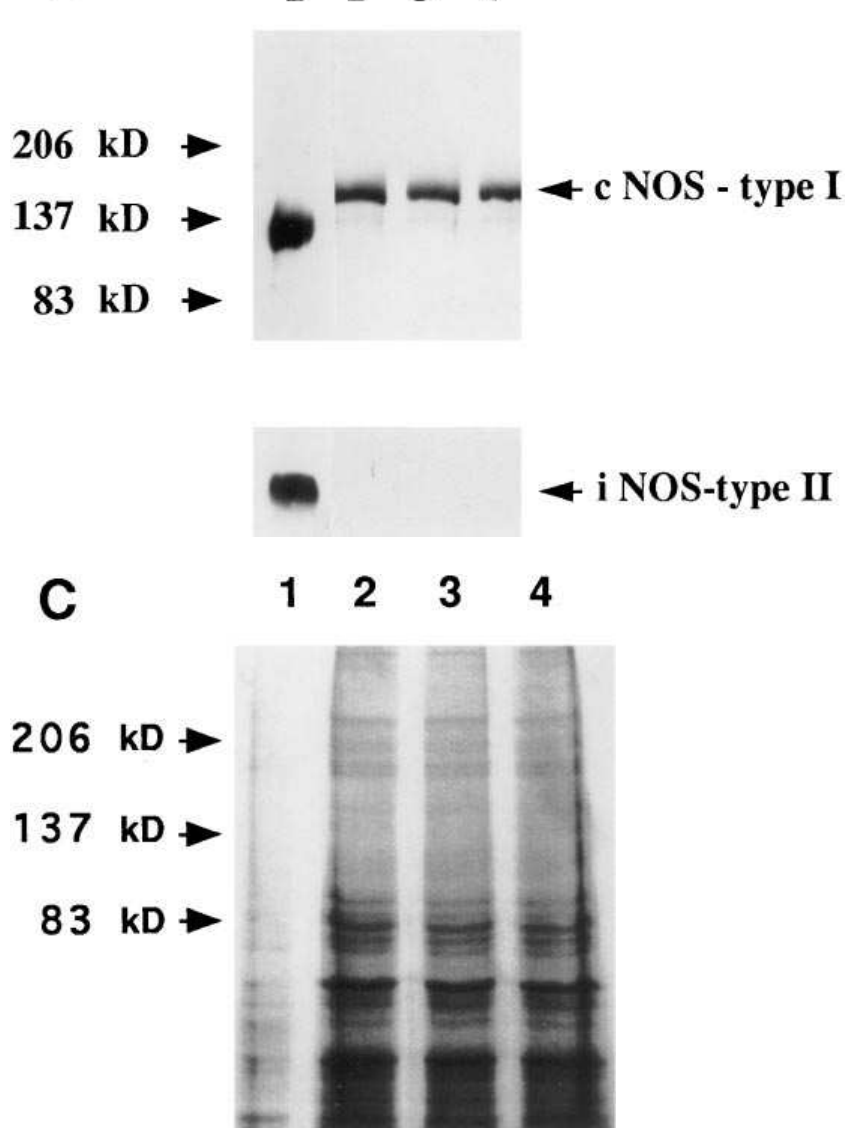

Figure 2. Characterization of the NOS type expressed by normal human keratinocytes after UV treatment. $(A)$ Reverse transcriptionPCR analysis of RNA isolated from keratinocytes. Cells were harvested $8 \mathrm{~h}$ after the last UV exposure. Total RNA was reverse transcribed with Moloney murine leukemia virus reverse transcriptase using oligo $(\mathrm{dT})_{15}$ as primer. The cDNA synthesized are PCR amplified with primer sets specific for brain cNOS, macrophage iNOS, and GAPDH as control. Lanes: $M, \mathrm{~F} \times 174 /$ Hae III-digest DNA; 1 , nonirradiated keratinocytes; 2, UVA-irradiated keratinocytes $(4 \times 300 \mathrm{~mJ} /$ $\left.\mathrm{cm}^{2}\right)$; 3, UVB-irradiated keratinocytes $\left(4 \times 50 \mathrm{~mJ} / \mathrm{cm}^{2}\right)$; and 4 , keratinocytes stimulated with LPS $(10 \mu \mathrm{g} / \mathrm{ml})$ and INF $\gamma(100 \mathrm{ng} / \mathrm{ml})$ for $48 \mathrm{~h}$. (B) Immunoblotting analysis of cNOS (brain type) and iNOS tion of tyrosinase) was estimated from the amount of $\left[{ }^{3} \mathrm{H}\right]_{2} \mathrm{O}$ released into the medium during the conversion of L- $\left[\right.$ ring $\left.-3^{\prime}-5^{\prime}-{ }^{3} \mathrm{H}\right]$ tyrosine to dihydroxyphenylalanine according to an adaptation (42) of the methods of Pomerantz (43) and Oikawa et al. (44), during the last day of treatment, as previously described (8). To avoid nonspecific incorporation of L- $\left[\right.$ ring $\left.-3^{\prime}-5^{\prime}-{ }^{3} \mathrm{H}\right]$ tyrosine into newly synthesized catecholamines by keratinocytes, the method was adapted for coculture experiments: $3 \mathrm{~h}$ after the last irradiation, keratinocytes (the upper compartment of the melanocyte-keratinocyte coculture) were removed before adding the $\mathrm{L}-\left[\right.$ ring $\left.-3^{\prime}-5^{\prime}-{ }^{3} \mathrm{H}\right]$ tyrosine into media during the next $21 \mathrm{~h}$.

Determination of melanin neosynthesis. Melanin synthesis was measured by the rate of incorporation of L-3,4-dihydroxyphenyl-[ $\left.3-{ }^{14} \mathrm{C}\right]$ alanine into newly synthesized melanins (45) during the last $24 \mathrm{~h}$ of melanocytes treatment as previously described (8).

\section{Reverse transcription-PCR analysis}

Total RNA from normal human keratinocytes were prepared using the RNAble kit (Eurobio, Strasbourg, France). First strand cDNA was made from $5 \mu \mathrm{g}$ RNA using the Moloney murine leukemia virus reverse transcriptase and oligo $(\mathrm{dT})_{15}$ as primers (Promega, Charbonniéres, France). For amplification of the cDNA, the following gene-specific primers were used: the cNOS primer set corresponded to nucleotide 4327-4344 (5'-ACCCTGCAGGCCAAGAA-3') and (5'-GGAGCTGAAAACCTCAT-3'), which is complementary to nucleotides 4712-4729 of human brain NOS cDNA (46). The iNOS synthase primer set were sense (5'-ATGCCAGATGGCAGCATCAGA-3') and antisense ( $5^{\prime}$-ACTTCCTCCAGGATGTTGTA-3') (47). GAPDH primer set were from Clontech (Ozyme, France). The following cycling times and temperatures were used to amplify cNOS and iNOS transcripts: 35 cycles of $94^{\circ} \mathrm{C}$ for $45 \mathrm{~s}, 47^{\circ} \mathrm{C}$ for cNOS and $60^{\circ} \mathrm{C}$ for iNOS for $60 \mathrm{~s}$, and $72^{\circ} \mathrm{C}$ for $90 \mathrm{~s}$.

\section{Western blot analysis}

For NOS expression analysis, proteins (macrophages: $5 \mu \mathrm{g}$; keratinocytes: $20 \mu \mathrm{g}$ ) were separated by electrophoresis on $7.5 \%$ polyacrylamide gels, electrotransferred to nitrocellulose and developed for anti-brain cNOS or anti-iNOS immunoreactivity by the indirect immunoperoxidase method and enhanced chemiluminescence detection (Amersham Laboratories). Control macrophage cell lysate, antiiNOS and antibrain cNOS monoclonal antibodies were from Affiniti. For the analysis of the expression of the melanogenic enzymes expression, $15 \mu \mathrm{g}$ proteins were separated by electrophoresis on $10 \%$ polyacrylamide gels, electrotransferred to nitrocellulose, and developed for antityrosinase or anti-TRP-1 immunoreactivity by the indirect immunoperoxidase method and enhanced chemiluminescence detection (Amersham Laboratories). The rabbit anti-human tyrosinase used in this study was a kind gift of Dr. Rorsman (48). The rabbit anti-PEP1 antibody, which recognizes the $\mathrm{COOH}$ terminus of the brown protein (TRP-1) (49) was kindly provided by Dr. Hearing.

(macrophage type) in human keratinocytes. Cell extracts $(20 \mu \mathrm{g})$ were subjected to SDS-PAGE followed by immunoblotting analysis using $1 \mu \mathrm{g} / \mathrm{ml}$ antibrain cNOS monoclonal antibody and $0.5 \mu \mathrm{g} / \mathrm{ml}$ anti-iNOS monoclonal antibody. Lane 1, macrophage (positive control); lane 2, nonirradiated keratinocytes; lane 3, UVA-irradiated keratinocytes $\left(4 \times 300 \mathrm{~mJ} / \mathrm{cm}^{2}\right)$; and lane 4 , UVB-irradiated keratinocytes $\left(4 \times 50 \mathrm{~mJ} / \mathrm{cm}^{2}\right)$. A specific immunoreactivity of keratinocyte lysates was obtained with the antibrain cNOS antibody (arrow, $155 \mathrm{kD}$ ). (C) SDS-PAGE analysis of protein cell lysates. Protein amounts were estimated using the Bio-Rad protein assay reagent and BSA as standard. Macrophage extract $(5 \mu \mathrm{g})$ and keratinocyte extracts $(20 \mu \mathrm{g})$ were subjected to SDS-PAGE, followed by coomassie blue staining. Lane 1, macrophage; lane 2, nonirradiated keratinocytes; lane 3, UVA-irradiated keratinocytes $\left(4 \times 300 \mathrm{~mJ} / \mathrm{cm}^{2}\right)$; and lane 4 , UVBirradiated keratinocytes $\left(4 \times 50 \mathrm{~mJ} / \mathrm{cm}^{2}\right)$. 

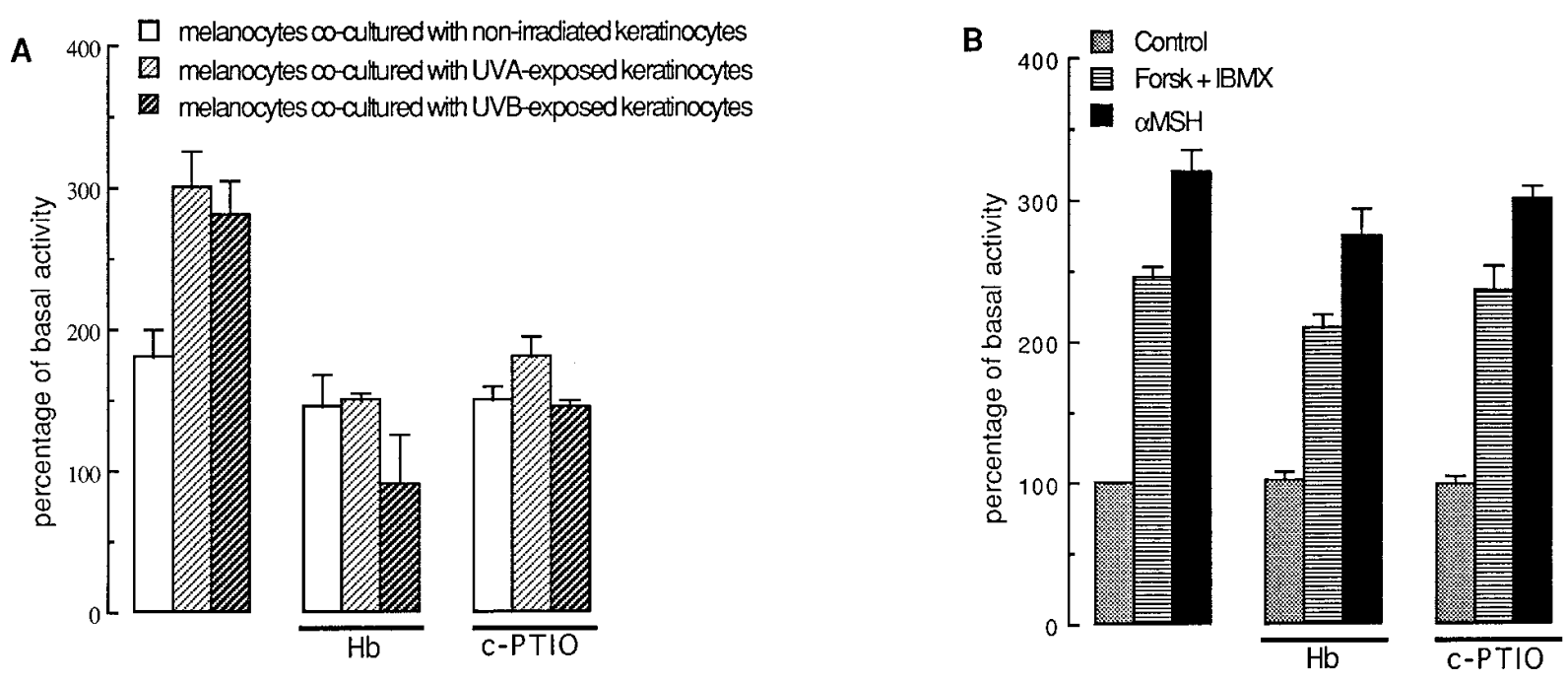

Figure 3. (A) Effect of conditioned media from UVA- and UVB-exposed keratinocytes on the tyrosinase activity of cultured melanocytes. Melanocytes were cocultured with keratinocytes as described in Methods. The keratinocyte compartment is exposed four times at 24-h intervals to the indicated dose and type of UV light. In some co-cultures, the NO scavengers, carboxy-PTIO $(20 \mu \mathrm{M})$, and $\mathrm{Hb}(1 \mathrm{mM})$ are added on the first day of phototreatment for the whole experiment period. Tyrosinase activity was determined using the in situ assay. Results are expressed as the percentage of basal activity of nonirradiated, separately cultured melanocytes. Each value represents the mean \pm SE of triplicates. Basal activity of tyrosinase: $42 \pm 3 \mu \mathrm{U} / \mathrm{mg}$ protein. $(B)$ Effect of NO scavengers on induced tyrosinase activity by cAMP elevating agents. Melanocytes were exposed to $60 \mu \mathrm{M}$ forskolin plus $200 \mu \mathrm{M}$ isobutyl methyl xanthin or $10 \mu \mathrm{M}$ [NLe $\mathrm{NL}^{4}$, $\left.\mathrm{Phe}^{7}\right] \alpha$-monocyte-stimulating hormone for $4 \mathrm{~d}$ in the absence or presence of $20 \mu \mathrm{M}$ carboxy-PTIO or $1 \mathrm{mM} \mathrm{Hb}$. Results are expressed as a percentage of the basal activity from nontreated melanocytes. Each value represents the mean $\pm \mathrm{SE}$ of triplicates of one representative experiment. Basal activity of tyrosinase: $36 \pm 1.2 \mu \mathrm{U} / \mathrm{mg}$ protein.

\section{Results}

Effects of $U V A$ and $U V B$ radiation on $N O$ production by keratinocytes. First, we studied the effect of UV radiation on NO production by keratinocytes (Fig. $1 A$ ). For this purpose, nitrite concentration was determined in keratinocyte-conditioned media after $4 \mathrm{~d}$ of culture. Nonirradiated keratinocytes produced a basal amount of nitrite ranging between 3 and $5 \mathrm{nmol} /$ million cells. The amount of NO released by UV-exposed keratinocytes was markedly increased by photo treatments: threefold for UVA and fourfold for UVB. Additionally, we observed a rapid and marked stimulation of NOS activity in keratinocytes at 30 min after single UVA or UVB exposures (Fig. 1 B). Further, this activity remained increased at $24 \mathrm{~h}$ after UV irradiation. These results show that UVA and UVB activate NOS, leading to an increased NO secretion. The study of the cofactors requirement for NOS activity showed a strong dependence upon calcium, suggesting the involvement of the cNOS rather than the iNOS in our system (data not shown).

Characterization of NOS in normal human keratinocytes required for $U V$-induced NO production. To formally identify the NOS isoform expressed in human keratinocytes, we performed reverse transcription-PCR assays on RNA extracted from cells exposed or not exposed to UV light (Fig. $2 A$ ). Using a primer set for specific cNOS type I (brain type), we amplified a 385-bp DNA fragment from the cDNA obtained in each condition. The sequence of the 385-bp PCR product was $100 \%$ homologue with the human brain NOS cDNA sequence (46). Using the iNOS-specific primer set on the same keratinocyte cDNA, no amplification could be detected. However, we detected the presence of iNOS cDNA (371 bp) in LPS/ INF $\gamma$-treated keratinocytes. Western blot performed with an anti-cNOS type I antibody (Fig. $2 B$, top) showed a single band of $155 \mathrm{kD}$ corresponding to cNOS that was not affected by the UV treatment. In macrophage extract, we detect a $135-\mathrm{kD}$ protein that is probably the iNOS type, since the antibody used in this study is known to cross-react with iNOS. With an antiiNOS type II antibody (Fig. $2 \mathrm{~B}$, bottom), we detected the iNOS type II (135 kD) from the macrophage extract. In keratinocytes, iNOS is absent even after UV treatment. A coomassie staining of the electrophoresis gel (Fig. $2 C$ ) revealed that each lane was loaded with the same protein amount. These results confirm the absence of iNOS in keratinocytes and suggest that the increase in NO production by UV-irradiated keratinocytes is due to an activation of cNOS.

Role of NO in the melanogenic effect of $U V$-irradiated keratinocyte conditioned media. Then we investigated the role of NO produced by keratinocytes in the melanogenic effect triggered by keratinocyte-conditioned media. Melanogenesis was estimated by measuring the activity of tyrosinase, the rate-limiting enzyme of melanin synthesis. Melanocytes cocultured with nonirradiated keratinocytes showed an $80 \%$ increase in tyrosinase activity compared with melanocytes alone (Fig. $3 A$ ). Moreover, the tyrosinase activity of melanocytes cocultured with UVA- or UVB-exposed keratinocytes was increased approximatively twofold compared with the tyrosinase activity of melanocytes cocultured with nonirradiated keratinocytes. This demonstrates that UV radiation induces keratinocytes to produce melanogenic agents. When the same experiment was performed in the presence of NO scavengers such as carboxyPTIO $(20 \mu \mathrm{M})$ or $\mathrm{Hb}(1 \mathrm{mM})$, the melanogenesis observed in the presence of nonexposed keratinocytes was decreased. Interestingly, the stimulation of melanogenesis induced by irradiated keratinocytes was completly blocked by NO scavengers. Next, in the aim of characterizing the specificity of the NO scavengers in inhibiting tyrosinase activity, we stimulated tyro- 

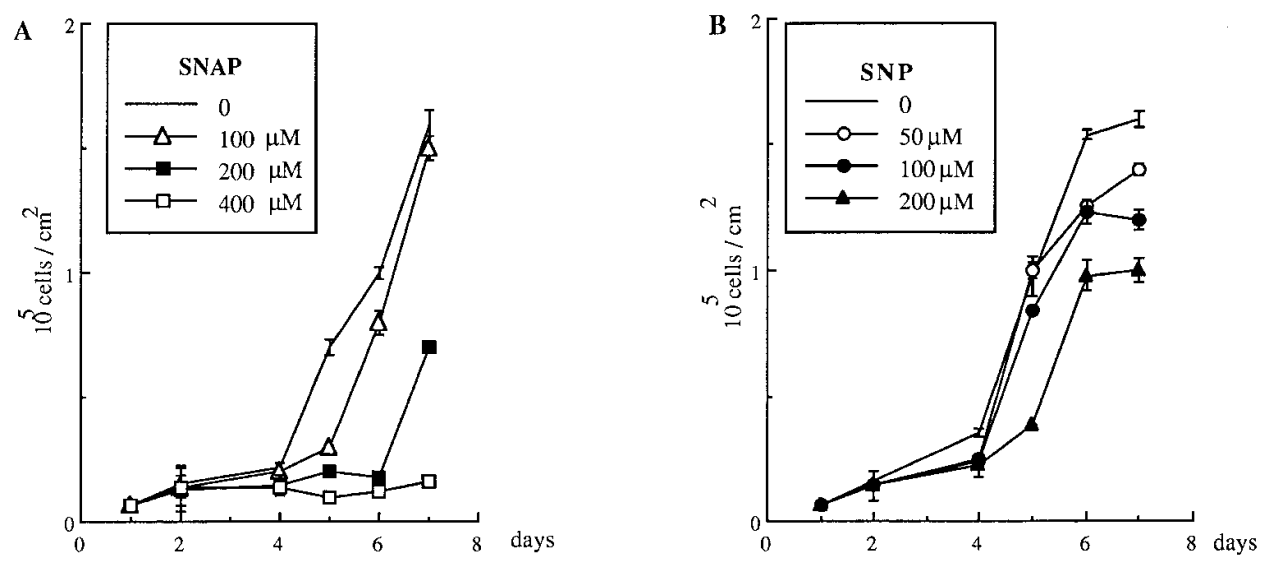

Figure 4. Antimitogenic effect of $\mathrm{NO}$ on normal human melanocytes. Melanocytes were grown in complete medium and incubated with increasing concentrations of NO donors $(S N A P$ or $S N P)$ for $7 \mathrm{~d}$. Melanocytes were refed each day. The cells remained attached throughout the experiment and excluded trypan blue. For each determination point, cells were trypsinized and counted with a Coulter counter. Each value represents the mean \pm SE of triplicates for each concentration.

sinase activity with cAMP-elevating agents in the absence or presence of these NO scavengers (Fig. $3 \mathrm{~B}$ ). Melanocytes treated for $96 \mathrm{~h}$ with $60 \mu \mathrm{M}$ forskolin plus $200 \mu \mathrm{M}$ isobutyl methyl xanthin or $10 \mu \mathrm{M}\left[\mathrm{NLe}^{4}, \mathrm{D}-\mathrm{Phe}^{7}\right] \alpha$-melanocyte-stimulating hormone presented, respectively, a 2.5- and a 3.5-fold increased tyrosinase activity. This increase was not significantly modified by the addition of NO scavengers, demonstrating the specificity of these products. Taken together, these results show that NO is involved in the melanogenic effect evoked by keratinocyte's conditioned media.
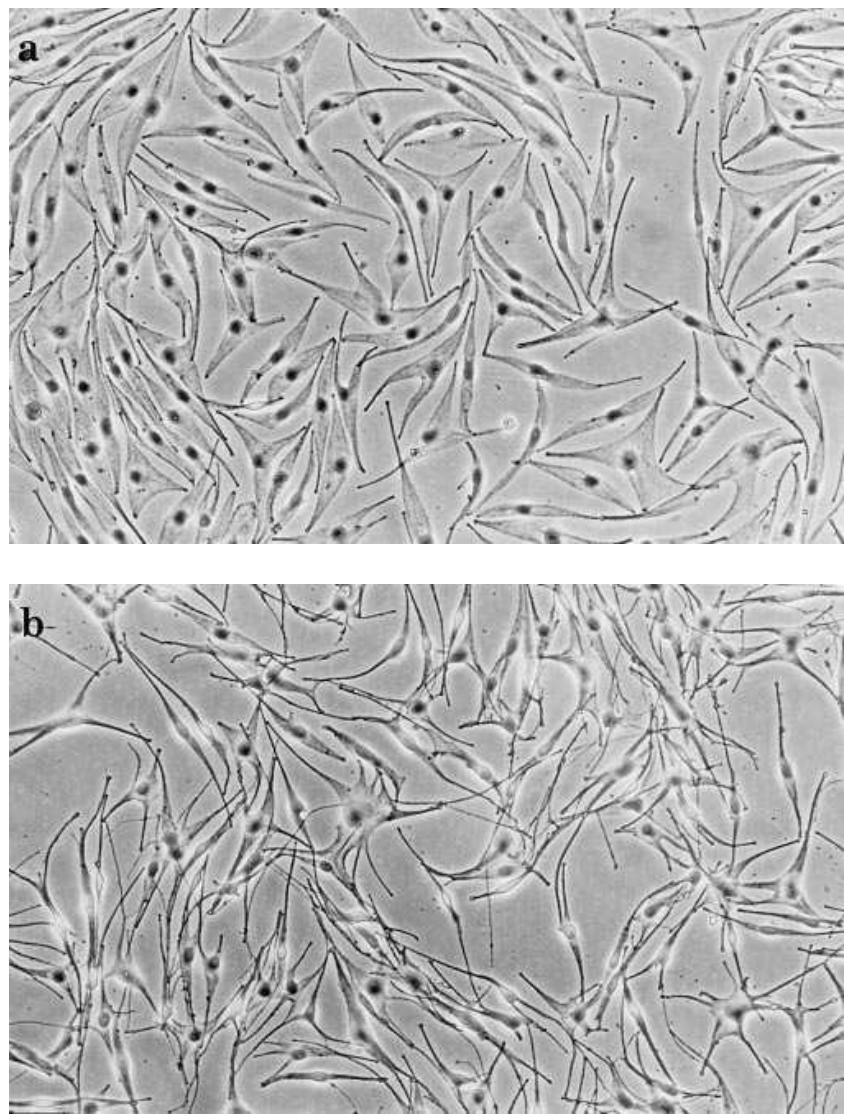

Figure 5. Effect of NO on melanocyte dendricity. Morphological appearance of nontreated melanocytes cultured in minimal medium $(a)$ or exposed to $200 \mu \mathrm{M}$ SNAP $(b)$ for $4 \mathrm{~d}$. Under phase contrast microscopy $(200 \times)$, a marked increase in number and length of dendrites is observed.
Effects of NO donors on human melanocytes dendricity, growth, and melanization. Since conditioned media from keratinocytes contains numerous agents that have been reported to influence melanocyte growth, dendricity, and melanization, we studied the effect of NO alone on these parameters. To investigate the effects of $\mathrm{NO}$ on melanocyte proliferation, cells were treated with increasing concentrations of two different chemical NO donors, SNAP and SNP. Growth measurements were performed by counting the cell number in triplicates, every day for a 7 -d period. High concentration of SNAP $(400 \mu \mathrm{M})$ totally inhibited melanocytes proliferation (Fig. $4 A$ ). After a 7 -d treatment, lower concentrations of SNAP $(200$ and $100 \mu \mathrm{M})$ were found to inhibit melanocyte proliferation by, respectively, 50 and $15 \%$. The second NO donor, SNP, was also found to decrease melanocyte growth in a dose-dependent manner (Fig. 4 B). However, the maximal inhibitory effect was $\sim 40 \%$ compared with total inhibition obtained with SNAP.

NO donors also affected melanocytes' morphology (Fig. 5). Indeed, melanocytes grown in minimal growth medium mostly appeared bi- or tri-polar with large cytosolic prolongations and enlarged cytoplasm. $4 \mathrm{~d}$ of treatment with $200 \mu \mathrm{M}$ SNAP induced a substantial raise in cell length (2.3-fold) and in the number of dendritic processes (Table I).

Next, we evaluated the effect of chemical NO donors on melanization. Melanocytes were treated for $96 \mathrm{~h}$ with NO donors, and then the tyrosinase activity and melanin neosynthesis were measured (Fig. $6 \mathrm{~A}$ ). Both NO donors markedly enhanced the tyrosinase activity in a dose-dependent manner to reach a threefold stimulation with $400 \mu \mathrm{M}$ SNAP and a fivefold stimulation with $200 \mu \mathrm{M}$ SNP. SNAP and SNP also increased melanin neosynthesis in a dose-dependent manner, as shown by the augmentation of $\left[{ }^{14} \mathrm{C}\right] \mathrm{DOPA}$ incorporation in

Table I. Effect of NO on Melanocyte Dendricity

\begin{tabular}{lcc}
\hline & Cell length & $\begin{array}{c}\text { Cells with at least } \\
\text { three dendrites }\end{array}$ \\
\hline & $\mu m$ & $\%$ \\
Nontreated melanocytes & $6.3 \pm 0.6$ & 28 \\
SNAP 200 $\mu \mathrm{M}$ & $14.3 \pm 0.9$ & 59
\end{tabular}

Melanocytes were cultured in the absence or presence of $200 \mu \mathrm{M}$ SNAP for $96 \mathrm{~h}$. Then cells were assayed for the different morphometric parameters indicated, as detailed in Methods. Results are means \pm SE of 100 determinations of one representative experiment. 

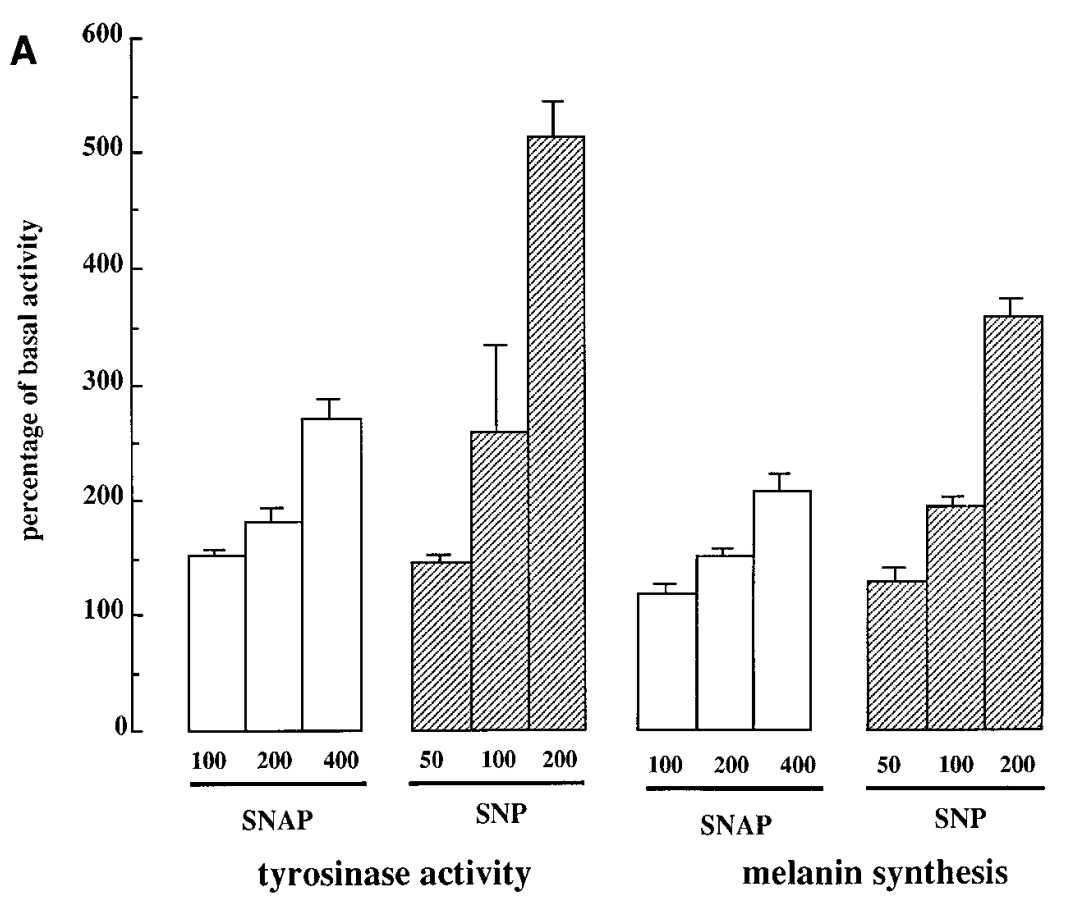

B
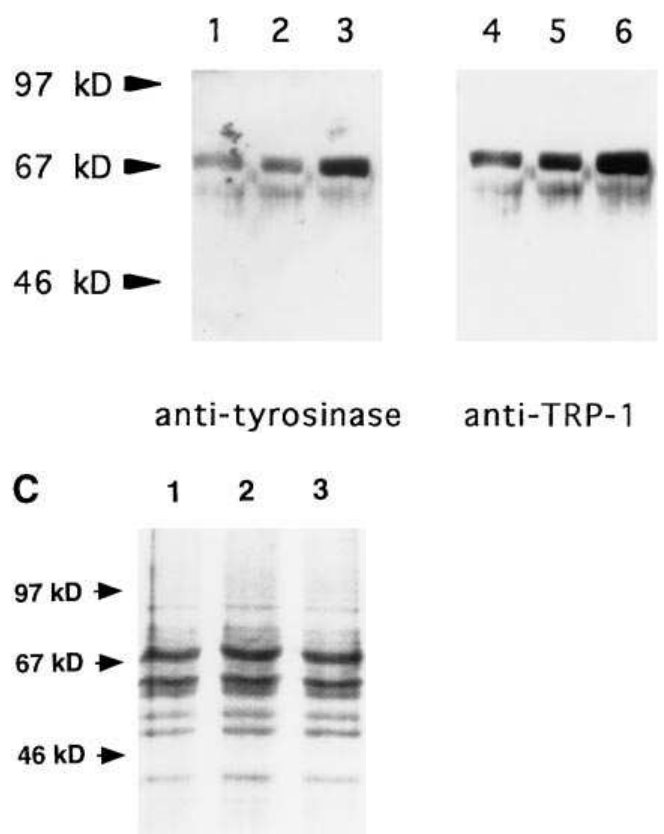

Figure 6. Effect of NO on melanocyte melanogenesis. (A) Effect of NO donors on tyrosinase activity and melanin synthesis of melanocytes. Melanocytes are exposed to SNAP $(100,200$, and $400 \mu \mathrm{M})$ or SNP $(50,100$, and $200 \mu \mathrm{M})$ for $4 \mathrm{~d}$. Results are expressed as a percentage of basal activity from control melanocytes. Each value represents the mean $\pm \mathrm{SE}$ of triplicates of one representative experiment. Basal activity of tyrosinase: $32 \pm 1.3 \mu \mathrm{U} / \mathrm{mg}$ protein. Basal incorporation of $\left[{ }^{14} \mathrm{C}\right] \mathrm{DOPA}: 293 \pm 18 \mathrm{nmol} / \mathrm{mg}$ protein. $(B)$ Immunoblotting analysis of tyrosinase and TRP-1 in human melanocytes exposed to SNAP. Cell extracts $(20 \mu \mathrm{g})$ are subjected to SDS-PAGE, followed by immunoblotting analysis using a rabbit anti-human tyrosinase antibody or a rabbit anti-TRP-1 antibody as indicated. Lanes 1 and 4, control melanocytes; lanes 2 and 5 , melanocytes exposed to $100 \mu \mathrm{M}$ SNAP for $4 \mathrm{~d}$; lanes 3 and 6 , melanocytes exposed to $200 \mu \mathrm{M}$ SNAP for $4 \mathrm{~d}$. (C) SDS-PAGE analysis of melanocyte extracts. Protein amounts were estimated using the Bio-Rad protein assay reagent. Cell extracts $(20 \mu \mathrm{g})$ were subjected to SDS-PAGE, followed by coomassie blue staining. Lanes 1 and 4, nontreated melanocytes; lanes 2 and 5, melanocytes exposed to $100 \mu \mathrm{M}$ SNAP for $4 \mathrm{~d}$; lanes 3 and 6 , melanocytes exposed to $200 \mu \mathrm{M}$ SNAP for $4 \mathrm{~d}$.

TCA-precipitable materials. The maximal stimulations with SNAP and SNP reached, respectively, 2- and 3.5-fold. To define if this effect was a consequence of a decreased proliferative rate induced by the SNAP treatment (200 $\mu$ M SNAP for $96 \mathrm{~h}$ ), we evaluated tyrosinase activity of melanocytes grown in minimal medium supplemented with $10 \%$ FCS (Table II). Under these conditions, melanocytes treated with $200 \mu \mathrm{M}$ of SNAP presented the same proliferative rate as untreated cells grown in minimal medium, while a stimulation of tyrosinase activity was still observed upon the SNAP treatment. This finding indicates that NO appears to be a specific element in the melanogenic process.

Then we studied the effect of SNAP on the expression of tyrosinase and TRP-1 in melanocytes (Fig. $6 \mathrm{~B}$ ). A coomassie blue staining of the SDS-PAGE (Fig. $6 C$ ) revealed that each lane of the electrophoresis gel was loaded with the same protein amounts. The dose-dependently increased melanin synthesis observed in melanocytes treated with SNAP was correlated with an increase in the amount of both tyrosinase and TRP-1.

\section{Discussion}

The present study shows that UV radiation, which is the main physiological stimulus for human skin pigmentation, activates human keratinocyte NOS with subsequent NO release in culture media. In addition, we demonstrate that NO, derived from chemical NO-donors as well as NO produced by UV- exposed keratinocytes, increases melanogenesis in human melanocytes, favoring the idea that NO is a keratinocyte-derived mediator involved in the control of melanogenesis.

Primary cultures of human keratinocytes have also been reported to respond to LPS/INF $\gamma$ or TNF $\alpha$ by producing NO, suggesting the expression of an inducible type of NOS in response to inflammatory stimuli $(24,25,47)$. In addition, UV radiation stimulates the release of proinflammatory cytokines (TNF $\alpha$, IL-1) by keratinocytes. It is tempting to propose that the effect of UV on NO production by keratinocytes is due to

Table II. Effect of NO on Melanocyte Tyrosinase Activity

\begin{tabular}{|c|c|c|c|c|}
\hline & \multicolumn{2}{|c|}{ Minimal medium } & \multicolumn{2}{|c|}{$\begin{array}{l}\text { Minimal medium } \\
\quad+10 \% \text { FCS }\end{array}$} \\
\hline & $\begin{array}{c}\text { Cell number } \\
\times 10^{-3} / \mathrm{cm}^{2}\end{array}$ & $\begin{array}{c}\text { Tyrosinase } \\
\text { activity } \\
\text { cpm } / 10^{6} \text { cells }\end{array}$ & $\begin{array}{c}\text { Cell number } \\
\times 10^{-3} / \mathrm{cm}^{2}\end{array}$ & $\begin{array}{c}\text { Tyrosinase } \\
\text { activity } \\
\text { cpm } / 10^{6} \text { cells }\end{array}$ \\
\hline Control melanocytes & $30 \pm 0.5$ & $32 \pm 0.3$ & $50 \pm 1$ & $57 \pm 0.1$ \\
\hline SNAP $200 \mu \mathrm{M}$ & $23 \pm 0.73$ & $76 \pm 0.9$ & $32 \pm 1.1$ & $84 \pm 0.9$ \\
\hline
\end{tabular}

Melanocytes were cultured in minimal medium or minimal medium supplemented with $10 \%$ FCS in the presence of 24-h-old solution of 200 $\mu \mathrm{M}$ SNAP (control) or $200 \mu \mathrm{M}$ SNAP for $96 \mathrm{~h}$. Tyrosinase activity was determined using the in situ assay. Results are means \pm SE of three determinations of one representative experiment. Basal activity of tyrosinase: $27 \pm 2 \mu \mathrm{U} / \mathrm{mg}$ protein. 
an increased expression of iNOS type II ensuing the release of these proinflammatory cytokines. However, the swift effect of UV on NOS activity suggests an activation of preexisting NOS, rather than new enzyme synthesis. Reverse transcription-PCR analysis and Western blot experiments show that keratinocytes expressed cNOS type I. The inducible NOS type II is exclusively detected in LPS/INF $\gamma$-treated keratinocytes. The absence of iNOS type II in UV-treated keratinocytes indicates that UV light activates the cNOS expressed in normal human keratinocytes. These observations are in agreement with recent reports indicating that normal human keratinocytes constitutively express the NOS type I (50) and that UVB exposure of the transformed keratinocyte cell line, SCC-13, enhanced NOS activity that was identified as a constitutive NO synthase regarding its strong dependence towards calcium (51). The mechanisms by which UV radiation activates cNOS remain puzzling. Nevertheless, it can be proposed that UV increases one of the cofactors involved in cNOS regulation, such as calcium, NADPH, or tetrahydro-biopterin (6-BH4) required for the enzymatic activity. Interestingly, UV radiation has been reported to upregulate total biopterin levels in human epidermis (52). Particularly in keratinocytes, this elevation could result in cNOS activation. Further, it should be noticed that 6-BH4 cofactor plays an essential role in the control of the synthesis of L-tyrosine (53) and bind to activate tyrosinase (54). Since L-tyrosine constitutes the first substrate in the melanin synthesis cascade controlled by tyrosinase, 6-BH4 could also directly regulate melanogenesis.

Compelling data have been gathered indicating that NO is involved in various networks of paracrine regulation. For example, NO freely diffuses from endothelium to vascular smooth muscle cell and across neuronal synapses to evoke a biological response (29). During the pigmentation process, melanocytes and keratinocytes closely interact within the epidermal-melanin unit. Therefore, NO produced by UV-irradiated keratinocytes could act as an intercellular mediator to regulate the melanogenesis of neighboring melanocytes. Indeed, melanogenic effects of conditioned media from both UVA/UVBexposed keratinocytes are markedly impaired by NO scavengers. However, the melanogenic effect of control keratinocytes is weakly affected by NO scavengers. These results indicate that NO plays a pivotal role in UV-induced melanogenesis. Recent reports indicate that keratinocytes produce melanogenic agents such as ET-1 (18), GM-CSF (19), and $\alpha$-melanocyte-stimulating hormone (55). Hence, NO could exert its melanogenic effects indirectly through the release of these melanogenic agents by keratinocytes. This hypothesis has been ruled out since melanocytes responded to chemical NO donors by increasing tyrosinase activity and melanin neosynthesis. The activation of melanogenesis, the induction of tyrosinase and TRP-1 expression by NO donors, and the inhibition of melanogenic effect of conditionated media from UV-exposed keratinocytes by NO scavengers converge to demonstrate that $\mathrm{NO}$ by itself is a potent stimulator of melanocyte melanogenesis and strengthen the pivotal role of NO as paracrine mediator in UV-induced skin pigmentation. Moreover, we have previously demonstrated that $\mathrm{UV}$ radiation upregulates tyrosinase and TRP-1 levels in human melanocytes $(8,32)$. Our new data raise the hypothesis that in vivo, $\mathrm{NO}$ derived from irradiated keratinocytes would potentiate the induction of tyrosinase and TRP-1 expression by UV.

In vivo, within $48 \mathrm{~h}$ after UV exposure, the epidermal me- lanocytes undergo cell proliferation with increased levels of tyrosinase activity $(56,57)$. This phenomenon is accompanied by an arborization and an increased length of dendritic processes, followed by enhanced synthesis and transfer of melanosomes to keratinocytes through the dendrites $(58,59)$. In vitro studies have demonstrated that UV radiation directly induces dendricity and melanin production in melanocytes. Nevertheless, the UV-induced proliferation observed in vivo was not present in vitro $(7,8)$. Similarly, NO donors induced melanocyte dendricity and inhibited the proliferation of cultured human melanocytes. This inhibition was not related to a cytotoxic effect since cells appeared healthy, without apparent evidence of damage and excluded trypan blue. In fact, the antiproliferative effect of NO is likely due to a stimulation of cell differentiation as it has been described in pheochromocytoma PC12 cells (60). Our data concerning the induction of melanocyte differentiation, in terms of dendricity and melanogenesis, support this concept.

On the other hand, ET-1, another UVB-induced keratinocyte-derived factor stimulates both proliferation and melanization of melanocytes (20). These observations strengthen the concept that several factors which may have opposite effects contribute to the final regulation of melanocytes growth and differentiation in vivo (15).

In summary, our data demonstrate that UV increases NO production by activation of cNOS keratinocytes and that NO is involved in the network of paracrine regulation triggered by UV, leading to the stimulation of skin pigmentation. This hypothesis remains to be confirmed in animal models and in human skin. Nevertheless, this study provides meaningful clues concerning the mechanism underlying UV-induced pigmentation of human skin and facilitates our fundamental understanding of melanogenesis.

\section{Acknowledgments}

The authors thank Dr. Rorsman and Dr. Hearing for their generous gifts of human tyrosinase antibody and TRP-1 antibody. We are very grateful to N. Biagoli for her precious help in cell culture and to W. Massabni for his constant technical assistance. We wish to thank Dr. R. Buscà for critical reading of this manuscript as well as A. Dalles, A. Grima, and C. Minghelli for illustration work. We also want to thank Dr. Bécherel for helpful discussions.

This work was supported by the Association pour la Recherche sur le Cancer (grant 6104), Fondation de France, Fondation pour la Recherche Médicale, Institut National de la Santé et de la Recherche Médicale, and l'Université de Nice Sophia-Antipolis.

\section{References}

1. Fitzpatrick, T.D., G. Szabo, M. Seiji, and W.C. Quevedo. 1979. Biology of the melanin pigmentary system. In Biology of the Melanocyte System. T. Fitzpatrick, editor. McGraw Hill Book Co., New York. 131-163.

2. DeLuca, M., F. D’Anna, S. Bondanza, A. Franzi Tito, and R. Cancedda. 1988. Human epithelial cells induce human melanocyte growth in vitro but only skin keratinocytes regulate its proper differentiation in the absence of dermis. $J$. Cell Biol. 107:1919-1926.

3. Yaar, M., and B.A. Gilchrest. 1991. Human melanocyte growth and differentiation: a decade of new data. J. Invest. Dermatol. 97:611-617.

4. Lacour, J.P., P.R. Gordon, M. Eller, J. Bhawan, and B.A. Gilchrest. 1992. Cytoskeletal events underlying dendrite formation by cultured pigment cells. $J$. Cell Physiol. 15:287-299.

5. Donatien, P., E. Surleve-Bazeille, A.J. Thody, and A. Taieb. 1993. Growth and differentiation of normal melanocytes in a cholera toxin and TPAfree, low serum medium and the influence of keratinocytes. Arch. Dermatol. Res. 285:385-392.

6. Rosen, C.F., Y. Seki, W. Farinelli, R.S. Stern, T.B. Fitzpatrick, M.A. 
Pathak, and R.W. Gange. 1987. A comparison of the melanocyte response to narrow band UVA and UVB exposure in vivo. J. Invest. Dermatol. 88:774-779.

7. Friedmann, P.S., and B.A. Gilchrest. 1987. Ultraviolet radiation directly induces pigment production by cultured human melanocytes. J. Cell Physiol. 133:88-94.

8. Aberdam, E., C. Roméro, and J.P. Ortonne. 1993. Repeated UVB irradiations do not have the same potential to promote stimulation of melanogenesis in cultured normal human melanocytes. J. Cell Sci. 106:1015-1022.

9. Eller, M.S., K. Ostrom, and B.A. Gilchrest. 1996. DNA damage enhances melanogenesis. Proc. Natl. Acad. Sci. USA. 93:1087-1092.

10. Kupper, T.S., A.O. Chua, P. Flood, J. McGuire, and U. Gubler. 1987. Interkeukin 1 gene expression in cultured human keratinocytes is augmented by ultraviolet irradiation. J. Clin. Invest. 80:430-436.

11. DeLeo, V., S. Scheide, J. Meshulam, D. Hanson, and A. Cardullo. 1988. Ultraviolet radiation alters choline phospholipid metabolism in human keratinocytes. J. Invest. Dermatol. 91:303-308.

12. Kupper, T.S., K. Min, P. Sehgal, H. Mizutani, N. Birchall, A. Ray, and L. May. 1989. Production of IL-6 by keratinocytes. Implications for epidermal inflammation and immunity. Ann. NY Acad. Sci. 557:454-464.

13. Bos, J.D., and M.L. Kapsenberg. 1993. The skin immune system: progress in cutaneous biology. Immunol Today. 14:75-80.

14. Kondo, S., T. Kono, D.N. Sauder, and R.C. McKenzie. 1993. IL-8 gene expression and production in human keratinocytes and their modulation by UVB. J. Invest. Dermatol. 101:690-694.

15. Gilchrest, B., H.Y. Park, M.S. Eller, and M. Yaar. 1996. Mechanisms of ultraviolet light-induced pigmentation. Photochem. Photobiol. 63:1-10.

16. Halaban, R., R. Langdon, N. Birshall, C. Cuono, G. Moellmann, and J. McGuire. 1988. Basic fibroblast growth factor from human keratinocytes is a natural mitogen for melanocytes. J. Cell Biol. 107:1611-1619.

17. Imokawa, G., G. Yada, and M. Miyagishi. 1992. Endothelins secreted from human keratinocytes are intrinsic mitogens for human melanocytes. $J$. Biol. Chem. 267:24675-24680.

18. Yohn, J.J., J.G. Morelli, S.J. Walchack, K.B. Rundell, D.A. Norris, and M.R. Zamora. 1993. Cultured human keranocytes synthesize and secrete endothelin-1. J. Invest. Dermatol. 100:23-26.

19. Imokawa, G., Y. Yada, M. Kimura, and N. Morisaki. 1996. Granulocyte/ macrophage colony-stimulating factor is an intrinsic keratinocyte-derived growth factor for human melanocytes in UVA-induce melanosis. Biochem. J. 313:625-631.

20. Yada, Y., K. Higuchi, and G. Imokawa. 1991. Effects of endothelins on signal transduction and proliferation in human melanocytes. J. Biol. Chem. 266: 18352-18357.

21. Moncada, S., R.M. Palmer, and E.A. Higgs. 1991. Nitric oxide: physiology, pathophysiology, and pharmacology. Pharmacol. Rev. 43:109-140.

22. Schmidt, H.H.H.W., and U. Walter. 1994. NO at work. Cell. 78:919-925.

23. Joshi, M. J Strandhoy, and W.L. White. 1996. Nitric Oxide synthase activity is up-regulated in melanoma cell lines: a potential mechanism for metastases formation. Melanoma Res. 6:121-126.

24. Heck, D.E., D.L. Laskin, C.R. Gardner, and J.D. Laskin. 1992. Epidermal growth factor suppresses nitric oxide and hydrogen peroxide production by keratinocytes. Potential role for nitric oxide in the regulation of wound healing. J. Biol. Chem. 267:21277-21280.

25. Bécherel, P.A., M.D. Mossalayi, F. Ouaaz, L. Le Goff, B. Dugas, N. Paul-Eugene, C. Frances, O. Chosidow, E. Kilchherr, J.J. Guillosson, et al. 1994. Involvement of cyclic AMP and nitric oxide in immunoglobulin E-dependent activation of $\mathrm{Fc} \in \mathrm{RII} / \mathrm{CD} 23$ + normal human keratinocytes. J. Clin. Invest. 93:2275-2279.

26. Warren, J.B. 1994. Nitric oxide and human skin blood flow responses to acetylcholine and ultraviolet light. FASEB J. 8:247-251.

27. Forstermann, U., H.H. Schmidt, J.S. Pollock, H. Sheng, J.A. Mitchell, T.D. Warner, M. Nakane, and F. Murad. 1991. Isoforms of nitric oxide synthase. Characterization and purification from different cell types. Biochem. Pharmacol. 42:1849-1857.

28. Forstermann, U., E.I. Closs, J.S. Pollock, P. Schwartz, J. Gath, and H. Kleinert. 1994. Nitric oxide synthase isozymes. Characterization, purification, molecular cloning, and functions. Hypertension (Dallas). 23:1121-1131.

29. Knowles, R.G., and S. Moncada. 1994. Nitric oxide synthases in mammals. Biochem. J. 298:249-258.

30. Bredt, D.S., and S.H. Snyder. 1994. Nitric oxide: a physiologic messenger molecule. Annu. Rev. Biochem. 63:175-195.

31. Nathan, C., and G.W. Xie. 1994. Nitric oxide synthases: roles, tolls, and controls. Cell. 78:915-916.

32. Roméro, C., E. Aberdam, C. Larnier, and J.P. Ortonne. 1994. Retinoic acid as modulator of UVB-induced melanocyte differentiation. Involvement of the melanogenic enzymes expression. J. Cell Sci. 107:1095-1103.

33. Englaro, W., R. Rezzonico, M. Durand-Clément, D. Lallemand, J.P. Ortonne, and R. Ballotti. 1995. Mitogen-activated protein kinase pathway and
AP-1 are activated during cAMP-induced melanogenesis in B-16 melanoma cells. J. Biol. Chem. 270:24315-24320.

34. Eisinger, M., and O. Marko. 1982. Selective proliferation of normal human melanocytes in vitro in presence of phorbol ester and cholera toxin. Proc. Natl. Acad. Sci. USA. 79:2018-2022.

35. Rheinwald, J.G., and H. Green. 1975. Serial cultivation of strains of human epidermal keratinocytes: the formation of keratinizing colonies from single cells. Cell. 6:331-344.

36. Boyce, S.T., and R.G. Ham. 1983. Calcium-regulated differentiation of normal human epidermal keratinocytes in chemically defined clonal culture and serum-free culture. J. Invest. Dermatol. 81:33-40.

37. O'Keefe, E.J., and M.L. Chiu. 1988. Stimulation of thymidine incorporation in keratinocytes by insulin, epidermal growth factor, and placental extract: comparison with cell number to assess growth. J. Invest. Dermatol. 90:2-7.

38. Jeanmougin, M. 1983. Photodermatoses et photoprotection. Roche, editor. Deltacom, Paris. $17-18$.

39. Wang, R., A. Ghahary, Y.J. Shen, P.G. Scott, and E.E. Tredget. 1996. Human dermal fibroblasts produce nitric oxide and express both constitutive and inducible nitric oxide synthase isoforms. J. Invest. Dermatol. 106:419-427.

40. Brouet, I., and H. Ohshima. 1995. Curcumin, an anti-tumour promoter and anti-inflammatory agent, inhibits induction of nitric oxide synthase in activated macrophages. Biochem. Biophys. Res. Commun. 206:533-540.

41. Evans, T., A. Carpenter, and J. Cohen. 1992. Purification of a distinctive form of endotoxin-induced nitric oxide synthase from rat liver. Proc. Natl. Acad. Sci. USA. 89:5361-5365.

42. Lotan, R., and D. Lotan. 1980. Stimulation of melanogenesis in a human melanoma cell line by retinoids. Cancer Res. 40:3345-3350.

43. Pomerantz, S. 1969. L-tyrosine $3,5-{ }^{3} \mathrm{H}$ assay for tyrosinase development of skin of newborn hamsters. Science (Wash. DC). 164:838-839.

44. Oikawa, A.M., M. Nakayasu, M. Nohara, and T.T. Tchen. 1972. Fate of $\mathrm{L}-\left(3,5-{ }^{3} \mathrm{H}\right)$ tyrosine in cell-free extracts and tissue cultured of melanoma cells. A new assay method for tyrosinase in living cells. Biochim. Biophys. Acta. 548-557.

45. Hearing, V.J., and T.M. Ekel. 1976. Mammalian tyrosinase. A comparison of tyrosine hydroxylation and melanin formation. Biochem. J. 157:549-557.

46. Nakane, M., H. Schmidt, J.S. Pollock, U. Fostermann, and F. Murad. 1993. Cloned human brain nitric oxide synthase is higly expressed in skeletal muscle. FEBS Lett. 316:175-180.

47. Bécherel, P.A., L. Le Goff, S. Ktorza, F. Ouaaz, J.M. Mencia-Huerta, B. Dugas, P. Debre, M.D. Mossalayi, and M. Arock. 1995. Interleukin-10 inhibits IgE-mediated nitric oxide synthase induction and cytokine synthesis in normal human keratinocytes. Eur. J. Immunol. 25:2992-2995.

48. Wittbjer, A., G. Odh, E. Rosengren, and H. Rorsman. 1991. A sensitive tyrosinase method for human skin. Acta Dermato-Venereol. 71:399-402.

49. Jimenez, M., K. Tsukamoto, and V.H. Hearing. 1991. Tyrosinases from two different loci are expressed by normal and transformed melanocytes. $J$. Biol. Chem. 266:1147-1156.

50. Baudouin, J.E., and P. Tachon. 1996. Constitutive nitric oxide synthase is present in normal human keratinocytes. J. Invest. Dermatol. 106:428-431.

51. Deliconstantinos, G., V. Villotiou, and J.C. Stravrides. 1995. Release by ultraviolet $\mathrm{B}$ radiation of nitric oxide from human keratinocytes: a potential role for nitric oxide in erythema production. British J. Pharmacol. 114:1257-1265.

52. Schulz-Douglas, V., K.U. Schallreuter, J.M. Wood, C. Körner, N.L. Lindsey, and E.R. Werner. 1996. Epidermal tetrahydrobiopterins promote de novo melanogenesis. J. Invest. Dermatol. 106:884. (Abstr.)

53. Schallreuter, K.U., J.M. Wood, M.R. Pittelkow, M. Gutlich, K.R Lemke, W. Rold, N.N. Swanson, K. Hitzemann, and I. Ziegler. 1994. Regulation of melanin biosynthesis in the human epidermis by tetrahydrobiopterin. Science (Wash. DC). 263:1444-1446.

54. Wood, J.M., K.U. Schallreuter-Wood, N.J. Lindsey, S. Callagan, and M.L. Gardner. 1995. A specific tetrahydrobiopterin binding domain on tyrosinase controls melanogenesis. Biochem. Biophys. Res. Commun. 206:480-485.

55. Schauer, E., F. Trautinger, A. Köck, A. Scharwz, and T.A. Lugar. 1994 Proopiomelanocortin-derived peptides are synthesized and released by human keratinocytes. J. Clin. Invest. 93:2258-2262.

56. Beitner, H. 1988. Immediate pigment darkening reaction. Photodermatology. 5:96-100.

57. Libow, L.F., S. Scheide, and V.A. DeLeo. 1988. Ultraviolet radiation acts as an independent mitogen for normal human melanocytes in culture. Pigm. Cell Res. 1:397-401.

58. Jimbow, K., M.A. Pathak, G. Szabo, and T.B. Fitzpatrick. 1974. Ultrastructural changes in human skin melanocytes after ultraviolet radiation. N.A In Pathak, L.D. Harber, M. Seiji, A. Kukita, and A. Sunlight, editors. Tokyo University Press, Tokyo. 192-215.

59. Megaw, J.M., and L.A. Drake. 1986. Drug and chemical toxicology. In Photobiology of the Skin and Eye. Vol. 5. Drug Chem. Toxicol.

60. Peunova, N., and G. Enikolopov. 1995. Amplification of calcium induced gene transcription by nitric oxide in neuronal cells. Nature (Lond.). 364:450-453. 